\title{
B-Lymphoma Development
}

National Cancer Institute

\section{Source}

National Cancer Institute. B-Lymphoma Development. NCI Thesaurus. Code C19386.

Malignant transformation and proliferation of B lymphocytes. 